\title{
Quantum States with a Positive Partial Transpose are Useful for Metrology
}

\author{
Géza Tóth ${ }^{1,2,3, *}$ and Tamás Vértesi ${ }^{4, \dagger}$ \\ ${ }^{1}$ Department of Theoretical Physics, University of the Basque Country UPV/EHU, P.O. Box 644, E-48080 Bilbao, Spain \\ ${ }^{2}$ IKERBASQUE, Basque Foundation for Science, E-48013 Bilbao, Spain \\ ${ }^{3}$ Wigner Research Centre for Physics, Hungarian Academy of Sciences, P.O. Box 49, H-1525 Budapest, Hungary \\ ${ }^{4}$ Institute for Nuclear Research, Hungarian Academy of Sciences, P.O. Box 51, H-4001 Debrecen, Hungary
}

(Received 27 September 2017; published 12 January 2018)

\begin{abstract}
We show that multipartite quantum states that have a positive partial transpose with respect to all bipartitions of the particles can outperform separable states in linear interferometers. We introduce a powerful iterative method to find such states. We present some examples for multipartite states and examine the scaling of the precision with the particle number. Some bipartite examples are also shown that possess an entanglement very robust to noise. We also discuss the relation of metrological usefulness to Bell inequality violation. We find that quantum states that do not violate any Bell inequality can outperform separable states metrologically. We present such states with a positive partial transpose, as well as with a nonpositive partial transpose.
\end{abstract}

DOI: 10.1103/PhysRevLett.120.020506

Introduction.-Entanglement lies at the heart of quantum mechanics and plays an important role in quantum information theory [1]. However, in spite of intensive research, many of the intriguing properties of entanglement are not fully understood. One of such puzzling facts is that, while entanglement is a sought-after resource, not all entangled states are useful for some particular quantum information processing application. For instance, in the Ekert protocol for quantum cryptography [2], entangled states that do not violate a Bell inequality are not useful. Moreover, maximally entangled singlets cannot be distilled from entangled quantum states that have a positive-semidefinite partial transpose (PPT). Such states, also called bound entangled, have been at the center of attention in quantum information science [3,4].

Recently, it has been realized that entangled states can be useful in very general metrological tasks in the sense that they make it possible to overcome the shot-noise limit in the precision of parameter estimation corresponding to classical interferometers [5-9]. Notably, separable states, i.e., states without entanglement, cannot overcome the classical limit. However, again, there are highly entangled states that are not useful for metrology [10].

The relation between the various subsets of entangled states have been studied for a long time. It has been conjectured by Peres that no bound entangled state violates a Bell inequality [11], which, after numerous attempts, has been recently refuted [12]. The search for counterexamples has been hindered by the fact that the conjecture is very close to be true. At this point, the questions arise: are there bound entangled states that are metrologically useful? Can states that do not violate any Bell inequality be metrologically useful? Finding such states numerically seems to be as easy as finding a needle in the haystack since we need to maximize a convex function over a convex set. There have been results concerning entanglement criteria with several quantum Fisher information terms detecting PPT entangled states as well as concerning the metrological usefulness of multipartite states that are not PPT with respect to all bipartions $[6,13,14]$. However, it is a famously hard open problem of quantum information theory whether states with only PPT entanglement can be useful for metrology [14].

In this Letter, we give an affirmative answer to the question above. We show that there are bound entangled states that outperform all separable states metrologically, as depicted in Fig. 1. Below, we summarize the four main contributions of this Letter.

(i) We present multiqubit quantum states that are metrologically useful, while having a positive partial transpose with respect to all bipartitions. In this way, we make sure that the metrological advantage compared to separable states cannot be attributed to the non-PPT bipartitions.

(ii) We also present several bipartite PPT states for dimensions from $3 \times 3$ to $12 \times 12$ that outperform separable states in quantum metrology. The metrological advantage of these states compared to separable states is very robust to

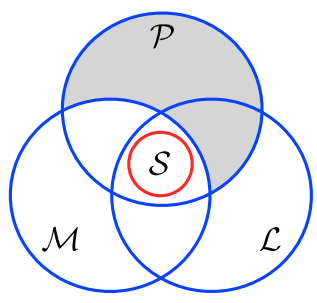

FIG. 1. Various convex sets of quantum states represented by circles: $(\mathcal{P})$ PPT states, $(\mathcal{M})$ states that are not useful for metrology, $(\mathcal{S})$ separable states, $(\mathcal{L})$ states with a local hidden variable model. (gray area) Metrologically useful PPT states. Such states are in $\mathcal{P} \backslash \mathcal{M}$, where $\backslash$ denotes the difference between two sets. 
noise. Thus, such states might be realized in experiments with photons or trapped cold ions (e.g., [15-18]).

(iii) We show an iterative method based on semidefinite programming (SDP) that can generate such states very efficiently. The method, starting from a given initial state, provides a series of PPT quantum states with a rapidly increasing metrological usefulness.

(iv) We now turn to the relation of metrological usefulness and other convex sets of quantum states. We show that quantum states with a local hidden variable model, i.e., not violating any Bell inequality, can be metrologically useful. We present such states with a positive as well as with a nonpositive partial transpose [14].

Quantum Fisher information.-Before discussing our main results, we review some of the fundamental relations of quantum metrology. A basic metrological task in a linear interferometer is estimating the small angle $\theta$ for a unitary dynamics $U_{\theta}=\exp (-i A \theta)$, where $A=\sum_{n=1}^{N} a^{(n)}, N$ is the particle number, and $a^{(n)}$ are single particle operators. The precision is limited by the Cramér-Rao bound as [19-21]

$$
(\Delta \theta)^{2} \geq 1 / \mathcal{F}_{Q}[\varrho, A],
$$

where the quantum Fisher information, a central quantity in quantum metrology, is defined by the formula [19]

$$
F_{Q}[\varrho, A]=2 \sum_{k, l} \frac{\left(\lambda_{k}-\lambda_{l}\right)^{2}}{\lambda_{k}+\lambda_{l}}|\langle k|A| l\rangle|^{2} .
$$

Here, $\lambda_{k}$ and $|k\rangle$ are the eigenvalues and eigenvectors, respectively, of the density matrix $Q$, which is used as a probe state for estimating $\theta$.

It has been shown that for separable multiqubit states, the quantum Fisher information, characterizing the maximal precision achievable by a quantum state, is bounded as [5]

$$
\mathcal{F}_{Q}\left[Q, J_{z}\right] \leq N,
$$

where $J_{z}=\sum_{n=1}^{N} j_{z}^{(n)}$, and $j_{z}^{(n)}$ are the single particle angular momentum components. Equation (3) can easily be generalized for qudits with a dimension $d>2$ and operators $A$ different from $J_{z}$. If $\mathcal{F}_{Q}[\varrho, A]$ is larger than the maximum reached by separable states, then $\varrho$ is useful for metrology. The maximum for separable states is given by $\sum_{n=1}^{N}\left[\lambda_{\max }\left(a^{(n)}\right)-\lambda_{\min }\left(a^{(n)}\right)\right]^{2}$, where $\lambda_{\min }\left(a^{(n)}\right)$ and $\lambda_{\max }\left(a^{(n)}\right)$ denote the minimum and maximum eigenvalues of $a^{(n)}$, respectively [22].

Main results.-Is Eq. (3) also valid for PPT states, i.e., multiqubit states, that are PPT with respect to all partitions? One could expect that this is the case since PPT states can only be weakly entangled, while they are highly mixed. The latter property hinders the violation of Eq. (3) since the Fisher information is convex, decreasing strongly after mixing quantum states.

Next, we present our first main result. We show that states with a positive partial transpose can still violate
Eq. (3) and its generalizations for $A \neq J_{z}$. Now, we give concrete examples, mentioning first only the main properties of the states found numerically.

Four-qubit state.-PPT with respect to all bipartitions, and with $A=J_{z}$.

Three-qubit state.-PPT with respect to all bipartitions. We consider operators different from $J_{z}$ and take $A=$ $j_{z}^{(1)}+j_{z}^{(2)}$.

Qubit-ququart bipartite PPT entangled state.-It is a three-qubit state for which only the 1:23 partition is PPT, while the other two bipartions are not PPT. Hence, the state has a higher value of quantum Fisher information than the three-qubit PPT state presented before. The three-qubit state can easily be transformed into a $2 \times 4$ bound entangled state, having the smallest dimensions in which PPT entanglement is possible.

Bipartite states of two qudits with equal dimension. $-d \times d$ states with $d=3,4, \ldots, 12$, with the operator

$$
A=\mathbb{1} \otimes D+D \otimes \mathbb{1}, \quad D=\operatorname{diag}(1,1, \ldots,-1,-1),
$$

where for even $d$ in the diagonal of $D$, there are $d / 21$ 's and $d / 2-1$ 's, and for odd $d$, there are $(d+1) / 21$ 's and $(d-1) / 2-1$ 's.

The quantum Fisher information of the states found, with other relevant properties, are summarized in Tables I and II. The density matrices of all the states are available in the Supplemental Material [22].

Maximization over PPT states.-We now describe the method that has been used to find the metrologically useful PPT states. Brute force maximization of the quantum Fisher information (2) for PPT states is extremely difficult since it is a convex function of the state. Hence, the maximum will be taken on the boundary of the set of PPT states, and no method can guarantee to find the global optimum.

We look for a simpler solution. We know that the error propagation formula gives the precision of estimating the parameter $\theta$ by measuring the expectation value of the operator $M$ as

$$
(\Delta \theta)^{2}=\frac{(\Delta M)^{2}}{\left|\partial_{\theta}\langle M\rangle\right|^{2}}=\frac{(\Delta M)^{2}}{\langle i[M, A]\rangle_{\varrho}^{2}} .
$$

TABLE I. Quantum Fisher information for PPT states found numerically in various systems. For each system, the maximum for separable states is shown. The robustness of the metrological usefulness of the states is also given, assuming white noise.

\begin{tabular}{lcccc}
\hline \hline System & $A$ & $\mathcal{F}_{Q}[\varrho, A]$ & $\mathcal{F}_{Q}^{(\text {sep })}$ & $p_{\text {white noise }}$ \\
\hline Four qubits & $J_{z}$ & 4.0088 & 4 & 0.0011 \\
Three qubits & $j_{z}^{(1)}+j_{z}^{(2)}$ & 2.0021 & 2 & 0.0005 \\
$2 \times 4$ (three qubits, & $j_{z}^{(1)}+j_{z}^{(2)}$ & 2.0033 & 2 & 0.0008 \\
$\quad$ only 1:23 is PPT) & & & & \\
\hline \hline
\end{tabular}


TABLE II. Quantum Fisher information for PPT states found numerically in two-qudit systems of local dimension $d$, where the maximum of the quantum Fisher information for separable states is 8. The operator $A$ is given in Eq. (4). For each state, the robustness of the metrological usefulness of the states is shown for white noise. A lower bound on the tolerated separable noise is also given.

\begin{tabular}{lccc}
\hline \hline$d$ & $\mathcal{F}_{Q}[\varrho, A]$ & $p_{\text {white noise }}$ & $p_{\text {noise }}^{\text {LB }}$ \\
\hline 3 & 8.0085 & 0.0006 & 0.0003 \\
4 & 9.3726 & 0.0817 & 0.0382 \\
5 & 9.3764 & 0.0960 & 0.0361 \\
6 & 10.1436 & 0.1236 & 0.0560 \\
7 & 10.1455 & 0.1377 & 0.0086 \\
8 & 10.6667 & 0.1504 & 0.0670 \\
9 & 10.6675 & 0.1631 & 0.0367 \\
10 & 11.0557 & 0.1695 & 0.0747 \\
11 & 11.0563 & 0.1807 & 0.0065 \\
12 & 11.3616 & 0.1840 & 0.0808 \\
\hline \hline
\end{tabular}

Based on Eq. (1), the quantity $1 /(\Delta \theta)^{2}$ provides a lower bound on the quantum Fisher information. Note that for some $M$, the bound is saturated $[21,35,36]$.

We now show that it is possible to obtain the quantum state minimizing Eq. (5).

Observation 1.-The minimum of the precision (5) for PPT states for a given operator $M$ can be obtained by a semidefinite program.

Proof.-Let us define first

$$
\begin{aligned}
& f_{M}(X, Y)=\min _{\varrho} \operatorname{Tr}\left(M^{2} \varrho\right), \\
& \text { such that } \varrho \geq 0, \varrho^{\mathrm{Tk}} \geq 0 \quad \text { for all } k, \operatorname{Tr}(\varrho)=1, \\
& \quad\langle i[M, A]\rangle=X, \quad \text { and } \quad\langle M\rangle=Y,
\end{aligned}
$$

where the optimization is carried out over a density matrix $Q$, which is PPT with respect to all bipartitions. Clearly, $f_{M}(X, Y)$ can be obtained via semidefinite programming. Note the important property that $f_{M}(X, Y)$ is convex in $X$ and $Y$ since the set of PPT states is a convex set. Then, the minimum of Eq. (5) for a given $M$ and for PPT states is

$$
(\Delta \theta)^{2}=\min _{X, Y} \frac{f_{M}(X, Y)-Y^{2}}{X^{2}},
$$

which needs an optimization over two real parameters.

If the measured operator $M$ is known, then Observation 1 provides a straightforward method to decide whether PPT states can outperform separable states. Based on Eqs. (1) and (3), we have to simply check whether $1 /(\Delta \theta)^{2} \leq N$ can be violated.

We now encounter the problem of how to obtain the optimal $M$ for which we can expect a violation of the bound corresponding to separable states. Next, we will present a very efficient solution to this problem [37].

Iterative Method.-To find a violation of the separability bound for the quantum Fisher information with PPT states of local Hilbert space dimension $d$, we use the following iterative procedure.

(1) Set $j=0$. Generate randomly a measurement operator $M$. Set $X$ to the average of the minimum and maximum eigenvalues of the expression $i[A, M]$.

(2) Compute $f_{M}(X, Y)$ from Eq. (6) for $Y=0$. This is a semidefinite program, which returns the optimal state $\varrho_{j}$. The optimal precision is $(\Delta \theta)_{Q_{j}}^{2}=\operatorname{Tr}\left(M^{2} \varrho_{j}\right) / X^{2}$ [c.f. Eq. (5)].

(3) Find the operator $M$ that achieves the highest $(\Delta \theta)_{Q_{j}}^{2}$ value for given $\varrho_{j}$. It is given by the symmetric logarithmic derivative [21]

$$
M=2 i \sum_{k, l} \frac{\lambda_{k}-\lambda_{l}}{\lambda_{k}+\lambda_{l}}|k\rangle\langle l|\langle k|A| l\rangle,
$$

where $\lambda_{k}$ and $|k\rangle$ are now the eigenvalues and eigenvectors, respectively, of $\varrho_{j}$. The quantum Fisher information of $\varrho_{j}$ can be obtained with $M$ as

$$
\mathcal{F}_{Q}\left[\varrho_{j}, A\right]=\operatorname{Tr}\left(M^{2} \varrho_{j}\right) .
$$

(4) Set $X=\langle i[M, A]\rangle$ and $j=j+1$.

(5) Repeat steps 2-4 until convergence of the objective value $\mathcal{F}_{Q}\left[Q_{j}, A\right]$ is reached.

Note that for the operator $M$ obtained in step 3, the relation $Y=\langle M\rangle=0$ holds. Hence, when at the next iteration, the algorithm reaches step 2 requiring $\langle M\rangle=0$ means that in the worst case, the same density matrix is found again as optimal. Typically, a better one is found, which implies $\mathcal{F}_{Q}\left[\varrho_{j}, A\right] \leq 1 /(\Delta \theta)_{Q_{j+1}}^{2}$. The latter inequality, together with the Cramér-Rao bound (1), yields $1 /(\Delta \theta)_{Q_{0}}^{2} \leq \mathcal{F}_{Q}\left[\varrho_{0}, A\right] \leq$ $1 /(\Delta \theta)_{Q_{1}}^{2} \leq \mathcal{F}_{Q}\left[\varrho_{1}, A\right] \leq \cdots$. Thus, the series $\mathcal{F}_{Q}\left[\varrho_{j}, A\right]$ never decreases.

The rapid convergence of the algorithm.-Our experience shows that the algorithm leads to a violation of the separable bound with 2-5 of trials in 10-20 iteration steps [38]. We plot the quantum Fisher information values of the density matrices obtained via the iterative algorithm for a concrete example in Fig. 2.

Robustness of the states obtained.-We examine how much the quantum states presented above can outperform separable states. The $12 \times 12$ bipartite state, referenced in Table II, shows a remarkably large violation of the bound corresponding to separability. The amount of violation can be characterized by the robustness of the metrological usefulness, i.e., the maximal amount of noise added for which the state performs still better than separable states. This can be obtained for white noise by direct calculation, while it can be bounded from below for PPT noise using semidefinite programming, see the Supplemental Material [22]. The robustness values obtained are given in Tables I and II. They indicate that some of our quantum states might be realized in the laboratory since they are resistant to the level of noise present in experiments. Note that the robustness of entanglement [39] is larger or equal to the robustness based on the metrological performance. 


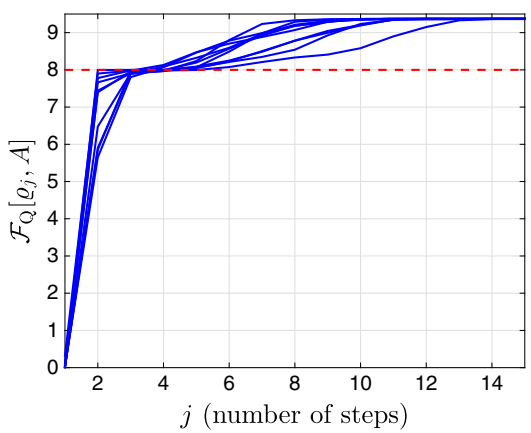

FIG. 2. Convergence to the optimal quantum Fisher information during the generation of the $4 \times 4$ bound entangled state, referred to also in Table II. The operator $A$ is given in Eq. (4). (solid) 10 attempts are shown. After 15 steps, the algorithm converged to the optimal value. (dashed) The maximal value of the quantum Fisher information for separable states.

Negativity.-So far, we have carried out an optimization for states that have a positive partial transpose for all bipartitions. The same algorithm can also be used if we relax this requirement to requiring that the smallest eigenvalue of the partial transposes is larger than $\lambda_{\min }$, where $\lambda_{\min }$ can now be negative. It is also possible to put a constraint on the negativity of the quantum state [40]. In the multipartite case, we can constrain the minimum of the bipartite negativities. To this end, we use a semidefinite program. The results can be found in the Supplemental Material [22].

Metrologically useful quantum states with a local hidden variable model. -As discussed in Ref. [14], it is an important question in entanglement theory whether states with another form of weak entanglement, i.e., entangled states with a local hidden variable model, can also be useful for metrology. We will answer the question affirmatively.

First, we describe an example with a positive partial transpose. For that, we consider the $2 \times 4$ state listed in Table I. We found that it is possible to construct numerically a local hidden variable model for the state using the algorithm of Refs. [41,42]. In Fig. 1, such states correspond to the set $\mathcal{P} \cap \mathcal{L} \backslash \mathcal{M}$, where $\cap$ denotes the intersection of two sets.

Next, we present non-PPT examples. Direct calculation shows that the two-qubit Werner state $p\left|\Psi^{-}\right\rangle\left\langle\Psi^{-}\right|+$ $(1-p) 1 / 4$, defined in Ref. [43], with $\left|\Psi^{-}\right\rangle=(|01\rangle-$ $|10\rangle) / \sqrt{2}$ for $p>0.3596$, is metrologically more useful than separable states, i.e., $\mathcal{F}_{Q}>2$. We considered the dynamics given by $A=j_{z} \otimes \mathbb{1}-\mathbb{1} \otimes j_{z}$. Such a state does not violate any Bell inequality for $p<0.6829$ using projective measurements $[44,45]$. In Fig. 1, metrologically useful Werner states with a local hidden variable model correspond to $\mathcal{L} \backslash \mathcal{P} \backslash \mathcal{M}$. A subset of the states above, i.e., Werner states for $p<5 / 12 \approx 0.4167$, do not violate any Bell inequality, even if positive operator valued measures (POVMs) are allowed [46].
Conclusions.-We showed that quantum states with a positive partial transpose can outperform separable states in the most general metrological task of estimating a parameter in linear interferometers. A powerful iterative method was presented for finding such states. We provided examples for multipartite systems, where all the partial transposes were positive. We also presented bipartite examples. Moreover, we presented PPT entangled states, as well as non-PPT entangled states, that do not violate any Bell inequality while they are still useful metrologically.

We thank I. Apellaniz, O. Gühne, M. Kleinmann, J. Siewert, and G. Vitagliano for discussions. We thank J. Kołodinski for drawing our attention to the reference [37]. We acknowledge the support of the EU (ERC Starting Grant No. 258647/GEDENTQOPT, COST Action CA15220, QuantERA CEBBEC), the Spanish Ministry of Economy, Industry and Competitiveness and the European Regional Development Fund FEDER through Grant No. FIS2015-67161-P (MINECO/FEDER, EU), the Basque Government (Grant No. IT986-16), and the National Research, Development and Innovation Office NKFIH (Grants No. K124351, No. K111734, and No. KH125096).

*toth@alumni.nd.edu; http://www.gtoth.eu †tvertesi@atomki.hu

[1] R. Horodecki, P. Horodecki, M. Horodecki, and K. Horodecki, Quantum entanglement, Rev. Mod. Phys. 81, 865 (2009); O. Gühne and G. Tóth, Entanglement detection, Phys. Rep. 474, 1 (2009).

[2] A. K. Ekert, Quantum Cryptography based on Bell's Theorem, Phys. Rev. Lett. 67, 661 (1991).

[3] A. Peres, Separability Criterion for Density Matrices, Phys. Rev. Lett. 77, 1413 (1996).

[4] P. Horodecki, Separability criterion and inseparable mixed states with positive partial transposition, Phys. Lett. A 232, 333 (1997).

[5] L. Pezzé and A. Smerzi, Entanglement, Nonlinear Dynamics, and the Heisenberg Limit, Phys. Rev. Lett. 102, 100401 (2009).

[6] P. Hyllus, W. Laskowski, R. Krischek, C. Schwemmer, W. Wieczorek, H. Weinfurter, L. Pezzé, and A. Smerzi, Fisher information and multiparticle entanglement, Phys. Rev. A 85, 022321 (2012); G. Tóth, Multipartite entanglement and high-precision metrology, Phys. Rev. A 85, 022322 (2012).

[7] B. Lücke, M. Scherer, J. Kruse, L. Pezzé, F. Deuretzbacher, P. Hyllus, J. Peise, W. Ertmer, J. Arlt, L. Santos, A. Smerzi, and C. Klempt, Twin matter waves for interferometry beyond the classical limit, Science 334, 773 (2011).

[8] R. Krischek, C. Schwemmer, W. Wieczorek, H. Weinfurter, P. Hyllus, L. Pezzé, and A. Smerzi, Useful Multiparticle Entanglement and Sub-Shot-Noise Sensitivity in Experimental Phase Estimation, Phys. Rev. Lett. 107, 080504 (2011).

[9] H. Strobel, W. Muessel, D. Linnemann, T. Zibold, D. B. Hume, L. Pezzé, A. Smerzi, and M. K. Oberthaler, Fisher 
information and entanglement of non-gaussian spin states, Science 345, 424 (2014).

[10] P. Hyllus, O. Gühne, and A. Smerzi, Not all pure entangled states are useful for sub-shot-noise interferometry, Phys. Rev. A 82, 012337 (2010).

[11] A. Peres, All the bell inequalities, Found. Phys. 29, 589 (1999).

[12] T. Vértesi and N. Brunner, Disproving the peres conjecture: Bell nonlocality from bipartite bound entanglement, Nat. Commun. 5, 5297 (2014).

[13] S. Altenburg, Ph.D. thesis, University of Siegen, 2017.

[14] L. Czekaj, A. Przysiężna, M. Horodecki, and P. Horodecki, Quantum metrology: Heisenberg limit with bound entanglement, Phys. Rev. A 92, 062303 (2015).

[15] N. Kiesel, C. Schmid, U. Weber, G. Tóth, O. Gühne, R. Ursin, and H. Weinfurter, Experimental Analysis of a FourQubit Photon Cluster State, Phys. Rev. Lett. 95, 210502 (2005).

[16] P. Walther, K. J. Resch, T. Rudolph, E. Schenck, H. Weinfurter, V. Vedral, M. Aspelmeyer, and A. Zeilinger, Experimental one-way quantum computing, Nature (London) 434, 169 (2005).

[17] H. Häffner, W. Hänsel, C. Roos, J. Benhelm, M. Chwalla, T. Körber, U. Rapol, M. Riebe, P. Schmidt, C. Becher, O. Gühne, W. Dür, and R. Blatt, Scalable multiparticle entanglement of trapped ions, Nature (London) 438, 643 (2005).

[18] D. Leibfried, E. Knill, S. Seidelin, J. Britton, R. B. Blakestad, J. Chiaverini, D. B. Hume, W. M. Itano, J. D. Jost, C. Langer, R. Ozeri, R. Reichle, and D. J. Wineland, Creation of a six-atom 'Schrödinger cat' state, Nature (London) 438, 639 (2005).

[19] C. Helstrom, Quantum Detection and Estimation Theory (Academic Press, New York, 1976); A. Holevo, Probabilistic and Statistical Aspects of Quantum Theory (NorthHolland, Amsterdam, 1982); S. L. Braunstein and C. M. Caves, Statistical Distance and the Geometry of Quantum States, Phys. Rev. Lett. 72, 3439 (1994); D. Petz, Quantum Information Theory and Quantum Statistics (Springer, Berlin, Heilderberg, 2008); S. L. Braunstein, C. M. Caves, and G. J. Milburn, Generalized uncertainty relations: Theory, examples, and lorentz invariance, Ann. Phys. (N.Y.) 247, 135 (1996).

[20] V. Giovannetti, S. Lloyd, and L. Maccone, Quantumenhanced measurements: Beating the standard quantum limit, Science 306, 1330 (2004); R. Demkowicz-Dobrzanski, M. Jarzyna, and J. Kolodynski, Chapter four - Quantum limits in optical interferometry, Prog. Opt. 60, 345 (2015); L. Pezze and A. Smerzi, Quantum theory of phase estimation, in Atom Interferometry Proceedings of International School of Physics Enrico Fermi, Course 188, Varenna, edited by G. Tino and M. Kasevich (IOS Press, Amsterdam, 2014) pp. 691-741; G. Tóth and I. Apellaniz, Quantum metrology from a quantum information science perspective, J. Phys. A 47, 424006 (2014).

[21] M. G. A. Paris, Quantum estimation for quantum technology, Int. J. Quantum. Inform. 07, 125 (2009).

[22] See Supplemental Material at http://link.aps.org/ supplemental/10.1103/PhysRevLett.120.020506 for additional results about maximizing the negativity and computing the robustness, as well as for the states found numerically, which includes Refs. [23-34].
[23] D. P. DiVincenzo, T. Mor, P. W. Shor, J. A. Smolin, and B. M. Terhal, Unextendible product bases, uncompletable product bases and bound entanglement, Commun. Math. Phys. 238, 379 (2003).

[24] H.-P. Breuer, Optimal Entanglement Criterion for Mixed Quantum States, Phys. Rev. Lett. 97, 080501 (2006).

[25] G. Tóth, T. Moroder, and O. Gühne, Evaluating Convex Roof Entanglement Measures, Phys. Rev. Lett. 114, 160501 (2015).

[26] G. Sentís, C. Eltschka, and J. Siewert, Quantitative bound entanglement in two-qutrit states, Phys. Rev. A 94, 020302 (2016).

[27] See the elin.m routine of the package QUBIT4MATLAB V5.5 available at http://www.mathworks.com/matlabcentral/ fileexchange. The 3.0 version of the package is described in G. Tóth, Comput. Phys. Commun. 179, 430 (2008). See also the CoRoNa package for the same aim at the same web address.

[28] G. Tóth and D. Petz, Extremal properties of the variance and the quantum fisher information, Phys. Rev. A 87, 032324 (2013).

[29] E. P. Wigner and M. M. Yanase, Information contents of distributions, Proc. Natl. Acad. Sci. U.S.A. 49, 910 (1963).

[30] R. Raussendorf and H. J. Briegel, A One-Way Quantum Computer, Phys. Rev. Lett. 86, 5188 (2001).

[31] O. Mandel, M. Greiner, A. Widera, T. Rom, T. W. Hansch, and I. Bloch, Controlled collisions for multi-particle entanglement of optically trapped atoms, Nature (London) 425, 937 (2003).

[32] V. Scarani, A. Acín, E. Schenck, and M. Aspelmeyer, Nonlocality of cluster states of qubits, Phys. Rev. A 71, 042325 (2005).

[33] O. Gühne, G. Tóth, P. Hyllus, and H. J. Briegel, Bell Inequalities for Graph States, Phys. Rev. Lett. 95, 120405 (2005).

[34] G. Tóth, O. Gühne, and H. J. Briegel, Two-setting bell inequalities for graph states, Phys. Rev. A 73, 022303 (2006).

[35] F. Fröwis, R. Schmied, and N. Gisin, Tighter quantum uncertainty relations following from a general probabilistic bound, Phys. Rev. A 92, 012102 (2015).

[36] I. Apellaniz, B. Lücke, J. Peise, C. Klempt, and G. Tóth, Detecting metrologically useful entanglement in the vicinity of dicke states, New J. Phys. 17, 083027 (2015).

[37] A method maximizing the QFI over noisy states, which does not use semidefinite programming, is given in K. Macieszczak, Quantum Fisher Information: Variational principle and simple iterative algorithm for its efficient computation, arXiv:1312.1356.

[38] For our calculations, we used MATLAB version 8.4.0 (R2014b) (The Mathworks Inc., Natick, Massachusetts, 2014). For semidefinite programming, we used the SeDuMi, SDTP3, and YALMIP packages. See L. Vandenberghe and S. Boyd, Semidefinite programming, SIAM Rev. 38, 49 (1996); J. F. Sturm, Implementation of interior point methods for mixed semidefinite and second order cone optimization problems, Optim. Methods Software 17, 1105 (2002); K. C. Toh, M. J. Todd, and R. H. Tutuncu, Sdpt3a matlab software package for semidefinite programming, Optim. Methods Software 11, 545 (1999); J. Löfberg, Yalmip: A toolbox for modeling and optimization in matlab, 
in Proceedings of the CACSD Conference, Taipei, Taiwan (IEEE, New York 2004).

[39] G. Vidal and R. Tarrach, Robustness of entanglement, Phys. Rev. A 59, 141 (1999).

[40] G. Vidal and R. F. Werner, Computable measure of entanglement, Phys. Rev. A 65, 032314 (2002).

[41] F. Hirsch, M. T. Quintino, T. Vértesi, M. F. Pusey, and N. Brunner, Algorithmic Construction of Local Hidden Variable Models for Entangled Quantum States, Phys. Rev. Lett. 117, 190402 (2016).

[42] D. Cavalcanti, L. Guerini, R. Rabelo, and P. Skrzypczyk, General Method for Constructing Local Hidden Variable Models for Entangled Quantum States, Phys. Rev. Lett. 117, 190401 (2016).
[43] R. F. Werner, Quantum states with einstein-podolsky-rosen correlations admitting a hidden-variable model, Phys. Rev. A 40, 4277 (1989).

[44] F. Hirsch, M. T. Quintino, T. Vértesi, M. Navascués, and N. Brunner, Better local hidden variable models for two-qubit Werner states and an upper bound on the Grothendieck constant $K_{G}(3)$, Quantum 1, 3 (2017).

[45] A. Acín, N. Gisin, and B. Toner, Grothendieck's constant and local models for noisy entangled quantum states, Phys. Rev. A 73, 062105 (2006).

[46] J. Barrett, Nonsequential positive-operator-valued measurements on entangled mixed states do not always violate a bell inequality, Phys. Rev. A 65, 042302 (2002). 